\title{
ATP oscillation caused by Gradual Entry of Substrates within Mitochondria
}

\author{
Taketoshi Hideshima ( $\nabla$ hidesima@obirin.ac.jp ) \\ J. F. Oberlin University \\ Mikie Nishimura \\ J. F. Oberlin University
}

\section{Research Article}

Keywords: mitochondria, oscillatory reaction, gradual entry, pyruvate, citric acid cycle, respiratory chain, ATP

Posted Date: March 19th, 2021

DOl: https://doi.org/10.21203/rs.3.rs-294605/v1

License: (1) This work is licensed under a Creative Commons Attribution 4.0 International License. Read Full License 


\section{Abstract}

The gradual entry of the substrate into enzyme solution causes the oscillatory reaction. We proved this by using dialysis membrane as a means of gradual entry of substrates ${ }^{7}$. It was considered that a suitable combination of permeation rate through membrane of the substrate and rate constants for catalytic reaction regulates the oscillation. It was suggested that such oscillatory reactions also should occur in actual living organisms. On the basis of this suggestion, we explored the oscillatory reaction within mitochondria, because many reactions in mitochondria also occur in the mediation of membrane. We found that the gradual entry of pyruvate together with ADP caused the oscillations of both NADH and ATP within mitochondria. Likewise, the gradual entry of $\mathrm{NAD}^{+}$and malate together with ADP caused the oscillations of NADH and of ATP. At the same time, pH oscillation within mitochondria was also observed. Besides the experiment with mitochondria, we also investigated oscillations of NADH and the other intermediates using dialysis membrane both in the citric acid cycle and in the respiratory chain. Putting these together, we concluded that the oscillatory reactions caused by the gradual entry of pyruvate occur continuously both in the citric acid cycle and in the respiratory chain and is taken over finally by the reaction of ATP synthase in the oxidative phosphorylation, inducing the oscillation of ATP. Furthermore, it was found that oscillations occurred without going through the citric acid cycle when $\mathrm{NAD}^{+}$and malate were used instead of pyruvate.

\section{Introduction}

Ordinary chemical reactions cause an increase in product and a decrease in reactant with progress of the time. Eventually, reactions attain equilibrium that both concentrations of product and reactant do not change anymore. However, it is well known that an oscillatory reaction or other kind of self-organization phenomenon appears in far-from-equilibrium situation ${ }^{1}$. The most famous chemical oscillator is nonlinear oscillatory reaction still known as the Belousov-Zhabotinsky (BZ) reaction which was demonstrated firstly by the Belousov ${ }^{2}$. An autocatalytic mechanism has been thought to be essential in BZ reaction ${ }^{3-4}$. On the other hand, in biochemistry, a glycolytic oscillation is the repetitive fluctuation of in the concentrations of metabolites, classically observed experimentally in yeast and muscle. The first observations of oscillatory behavior in glycolysis were made by Duysens and Amesz in $1957^{5}$. Glycolytic oscillation has been thought to be caused by allosteric enzymes ${ }^{6}$.

Apart from oscillations well known so far, we have discovered an oscillatory reaction of catalase induced by the gradual entry of hydrogen peroxide into enzyme solution ${ }^{7}$. As a means of the gradual entry of substrate, we have used means of the permeation of substrate through dialysis membrane, being semipermeable membrane. Ahead of the use of semipermeable membrane, we found an oscillatory reaction of alcohol dehydrogenase by using oil/water interface system ${ }^{8-9}$. Until now, we have observed oscillatory reactions of many kinds of enzymes, urease, acylase I, acetylcholine esterase, choline oxidase, lactate dehydrogenase, creatine kinase, et al., by using dialysis membrane ${ }^{10}$. Oscillatory reaction of catalase wrapped by liposome made of phospholipid was also observed ${ }^{11}$. Underlying cause of 
oscillatory reaction by enzyme is the gradual entry of substrate and a suitable combination of permeation rate through membrane of substrate and rate constants for catalytic reaction regulates the oscillation. In addition to the oscillatory reaction caused by enzyme, we have found calcium oscillation during the reaction between chondroitin sulfate and $\mathrm{Ca}^{2+}$ by using dialysis membrane ${ }^{12}$. Furthermore, we have observed calcium oscillation in the reaction between phospholipid and $\mathrm{Ca}^{2+}$ by using oil-water interface 10. Summarizing these results, the gradual entry of a substrate into reaction system has been thought to be essential for causing the oscillatory reaction. Based on these results, we thought it possible that similar oscillations occur in reactions which are concerned with naturally occurring biological membrane. So, we intended to employ naturally occurring biological membrane for investigating the oscillatory reaction in living organisms. As a material to explore the oscillation, we chose mitochondria in which ATP is produced as an energy source.

Aerobic organisms are able to capture a far greater proportion of the available free energy of respiratory substrate than anaerobic organisms. Reactions of respiratory chain take place inside mitochondria. Respiration is coupled to the generation of the higher energy intermediate, ATP, by oxidative phosphorylation. Mitochondria have an outer membrane and an inner membrane that is selectively permeable, enclosing a matrix within. Electrons flow through the respiratory chain passing through three large protein complexes: NADH-CoQ oxidoreductase (Complex I), CoQ-cytochrome c oxidoreductase (Complex III) and cytochrome c oxidase (Complex IV). The proton motive force drives a membranelocated ATP synthase that forms ATP in the presence of Pi + ADP. ATP synthase in embedded in the inner membrane, together with the respiratory complexes. Whether or not the oscillation of ATP happens really in the process of oxidative phosphorylation on the basis of our hypothesis that the gradual entry of substance causes oscillatory reaction? Elucidating this question is the purpose of this study.

\section{Results}

\section{Oscillations of NADH and ATP caused by gradual entry of pyruvate within mitochondria}

First of all, we investigated the NADH oscillation when pyruvate entered within mitochondria. When pyruvate passes across mitochondrial inner membrane, mitochondrial pyruvate carrier (MPC), which is protein embedded within membrane, facilitates the pass of pyruvate across membrane ${ }^{14-15}$. After a reaction of pyruvate with $\mathrm{NAD}^{+}$and $\mathrm{CoA}$ within inner membrane of mitochondria, reactions of citric acid cycle and respiratory chain proceed. In order to ascertain the existence of NADH oscillation in these processes, at first, isolated mitochondria were dispersed in the Tris-buffer solution $(2 \mathrm{~mL})$ containing sodium pyruvate in a cuvette and the time course of the fluorescence of NADH was measured at $340 \mathrm{~nm}$ (exciting light) and $460 \mathrm{~nm}$ (emission light). Figure 1a shows that the concentration of NADH decreased with oscillation at the condition of $50 \mathrm{mM}$ pyruvate. Further decreasing in pyruvate concentration led to disappearance of oscillation gradually.

Then, ADP, which was the substrate of ATP synthase, was also added to the solution of mitochondria and pyruvate. The time course of fluorescence intensity due to NADH is shown in Fig. 1b. NADH oscillation 
occurred in the same way even when ADP was added.

Further, in order to verify the generation and oscillation of ATP, $1 \mathrm{mM}$ luciferin $(0.1 \mathrm{~mL}), 1 \mathrm{mg} / \mathrm{mL}$ luciferase $(0.1 \mathrm{~mL})$ and $1 \mathrm{mM} \mathrm{Mg}^{2+}$ ion $(0.05 \mathrm{~mL})$ was added to the solution of mitochondria and pyruvate. Fluorescence was observed at the wave lengths of $550 \mathrm{~nm}$. The oscillation of ATP, however, was not observed at the same condition as those of Fig. $1 \mathrm{a}$ and $1 \mathrm{~b}$.

As shown in Fig. 1c, short-period oscillation of ATP (10 min 20min) was observed in the concentration range of $20 \mathrm{mM}$ or less of pyruvate at fixed concentration of ADP $(6.67 \mathrm{mM})$ after induction period. The phase diagram, which demonstrates the range of concentrations of pyruvate and ADP where oscillatory reaction happened, is shown in Fig. $1 \mathrm{~d}$.

In order to ascertain that florescence by NADH and ATP was due to which inside or outside of mitochondria, fluorescence was measured after removing mitochondria by centrifugation. The result showed that fluorescence was due to outer solution of mitochondria. NADH and ATP produced in mitochondria should flow out or flow in corresponding to the concentration difference between inside and outside of mitochondria membrane. Consequently, observed oscillations strongly support that the oscillations take place in mitochondria.

\section{Oscillations of NADH and ATP in the process via malate-aspartate shuttle}

NADH produced in glycolysis is also utilized in the respiratory chain. Although NADH is not possible to permeate through membrane, $\mathrm{NAD}^{+}$is possible to permeate through membrane and is converted to $\mathrm{NADH}$ by utilizing malate-aspartate shuttle ${ }^{13}$. So, we investigated the oscillation in the case of adding mitochondria to the solution containing $\mathrm{NAD}^{+}$and malate by measuring the fluorescence. The fluorescence of NADH was measured at $340 \mathrm{~nm}$ (exciting light) and $460 \mathrm{~nm}$ (emission light).

Figure $2 a$ shows that NADH oscillation occurred with a decrease in NADH concentration in a wide range of $\mathrm{NAD}^{+}$and malate in the absence of ADP supply. When ADP was added in this system, as shown in Fig. $2 \mathrm{~b}$ and $2 \mathrm{c}$, oscillations of both NADH and ATP with short period and small amplitude happened, being different from that of pyruvate solution. Furthermore, the oscillation lasted for long time and occurred in higher concentration of ADP compared to that caused by gradual entry of pyruvate. It was found that oscillation occurs without going through the process of citric acid cycle.

Then, we investigated the time course of $\mathrm{pH}$ within mitochondria for both systems of permeations of pyruvate and $\mathrm{NAD}^{+}$by using $\mathrm{pH}$-dependent fluorescent reagent. The results are shown in Fig. $2 \mathrm{~d}$ and $2 \mathrm{e}$. In both systems, pH oscillation was also observed as well as those of NADH and ATP.

In addition to the investigation with mitochondria, in order to verify an assumption that NADH and the other intermediates oscillations triggered by the gradual entry of first substrates (pyruvate or NADH) occur continuously in the citric acid cycle and the respiratory chain, we carried out the following experiments by using dialysis membrane. 


\section{Oscillations of respiratory chain intermediates using semipermeable membrane as a model of mitochondria membrane}

In addition to NADH produced by the citric acid cycle, NADH produced by glycolysis is also utilized in the respiratory chain. Although NADH is not able to permeate through mitochondrial membrane, NADH is produced from NAD ${ }^{+}$within matrix of mitochondria by malate-aspartate shuttle ${ }^{13}$. In order to prove an assumption that the gradual entry of NADH caused oscillatory reactions of intermediates existing in respiratory chain, a method using dialysis membrane was employed as a simple model of permeation through membrane.

At first, the direct reaction between NADH and coenzyme Q (CoQ) was investigated. NADH solution was put in upper phase of apparatus of Fig. 3, while liposome solution of L-a- dipalmitoyl phosphatidylcholine (DPPC) containing CoA was put in lower phase. Time course of the absorbance at $340 \mathrm{~nm}$, which is assigned to the absorption of NADH, was measured. As shown in Fig. 4a, direct reaction of NADH and CoQ occurred after an increase in the concentration of NADH accompanying the permeation of NADH across the membrane. Then the repetition of increase and decrease of absorbance occurred, i.e., oscillation was observed. The decrease in pyruvate concentration led to the oscillation with short period and small amplitude after that of long period and large amplitude.

Secondly, we added cytochrome $c$ and cytochrome $c$ reductase to lower phase. The reaction in lower phase is thought to be equivalent to that of complex III in respiratory chain. In this reaction, the electron of $\mathrm{NADH}$ is transferred to cytochrome $\mathrm{c}$ via CoQ. As the wavelength of the absorption of NADH and of reduced type of cytochrome $c$ is $340 \mathrm{~nm}$ and $550 \mathrm{~nm}$, respectively, the absorptions at $340 \mathrm{~nm}$ and $550 \mathrm{~nm}$ were measured at the same time. As shown in Fig. 4b, the oscillation of NADH appeared explicitly. Correspond to the oscillation of $\mathrm{NADH}$, the oscillation of cytochrome $\mathrm{c}$ with same period was observed. Two oscillations were synchronized.

Furthermore, small quantity of cytochrome c oxidase was also added to lower phase. This reaction is thought of corresponding to that of complex IV in respiratory chain. As shown in Fig. 4c, the addition of cytochrome $\mathrm{c}$ oxidase led to little change of oscillation pattern at the same concentration as cytochrome $c$ reductase. So, we reduced the concentration of cytochrome c reductase. As shown in Fig. 4d, when the concentration of cytochrome $\mathrm{c}$ oxidase equals to that of cytochrome $\mathrm{c}$ reductase, the oscillation with period of about $20 \mathrm{~h}$ occurred in both of NADH and cytochrome c. In greater quantity of cytochrome $\mathrm{c}$ oxidase than cytochrome $c$ reductase, distinct oscillation with short period appeared. We found that the oscillatory reactions of $\mathrm{NADH}$ and $\mathrm{CoQ}$ induced continuous oscillatory reactions for the respiratory chain.

For a reaction using semipermeable membrane, we only obtained the oscillation with longer-period compared to the result with mitochondria. This may be owing to the shortage of supply of oxygen from the air required for the reaction of cytochrome $c$ oxidase, because oxygen supply is hampered by the presence of upper phase sandwiched by dialysis membrane. Thus, in order to supply oxygen, to the system of Fig. 4c, $0.1 \mathrm{~mL}$ of $0.6 \%$ hydrogen peroxide was added to upper phase, while $0.1 \mathrm{~mL}$ of 
$0.1 \mathrm{mg} / \mathrm{mL}$ catalase to lower phase. It has been found already that this condition leads no oscillation of

oxygen ${ }^{7}$. The result is shown in Fig. 4e. It was found that the short-period oscillation appeared by the supply of oxygen. Further increasing the concentration of cytochrome c reductase shortens the period of oscillation. Oxygen supply seems to facilitate the reaction of cytochrome $c$ oxidase and to strengthen the oscillation.

\section{The consecutive oscillations of intermediates in citric acid cycle caused by the oscillatory reaction of pyruvate dehydrogenase due to gradual entry of pyruvate}

Pyruvate produced by glycolysis flows through mitochondrial membrane and is changed to acetyl-CoA by pyruvate dehydrogenase which catalyzes the reaction of pyruvate, $\mathrm{NAD}^{+}$and $\mathrm{CoA}$. When pyruvate passes across the mitochondrial inner membrane, mitochondrial pyruvate carrier (MPC), which is protein embedded within membrane, facilitates the pass of pyruvate across membrane ${ }^{8-9}$. Since the flow of pyruvate is also considered to be caused by the difference of electrochemical potential between the inside and the outside of mitochondrial inner membrane, we might assume for simplicity that semipermeable membrane is able to be employed instead of mitochondrial membrane.

At first, in apparatus of Fig. 3, we put pyruvate solution in upper phase, while pyruvate dehydrogenase, CoA and $\mathrm{NAD}^{+}$in lower phase, for measuring the formation of acetyl-CoA and NADH. Time course of the absorbance at $340 \mathrm{~nm}$ due to the absorption of NADH was measured.

As shown in Fig. 5a, when the concentration of pyruvate was increased at the fixed concentrations of $\mathrm{NAD}^{+}$and $\mathrm{CoA}$, the period of NADH oscillation became short. On the other hand, decreasing the concentration of $\mathrm{COA}$ at the fixed concentration of pyruvate and $\mathrm{NAD}^{+}$led to a stepwise increase in absorbance (Fig. 5b). From these results, it was considered that the oscillation of acetyl-CoA, which was another product of this reaction, also happened.

As it was found that the formation of acetyl-CoA was oscillatory reaction in the mediation of semipermeable membrane, we conceived that subsequent reactions in citric acid cycle oscillate similarly. Therefore, oxaloacetic acid, citrate synthase and aconitase were also added to lower phase. The absorbance of at both $340 \mathrm{~nm}$ and at $240 \mathrm{~nm}$, which were due to the absorptions of NADH and cis-aconitic acid, respectively, were measured at the same time.

As shown in Fig. 5c, not only the oscillation of NADH, but also that of cis-aconitic acid, the intermediate in the reaction of aconitase, was observed. When pyruvate concentration was small, the oscillations of NADH and cis-aconitic acid was restrained. However, as pyruvate concentration was increased, two oscillations were synchronized and the amplitude of cis-aconitic acid oscillation became large. Further increase of pyruvate led to short period and small amplitude oscillation of $\mathrm{NADH}$. The oscillation of $\mathrm{NADH}$ is thought to induce the oscillatory reaction of aconitase via that of citrate synthase. The reactions of citric synthase and of aconitase are those in first stage of citric acid cycle. As cis-aconitic acid is the 
intermediate of citric acid cycle, this result suggests that the oscillatory reaction will be passed down to remained reactions in citric acid cycle.

\section{Discussion}

We have already discovered the oscillatory reaction of enzyme caused by the gradual entry of substrate using dialysis membrane. By using this method, we studied the oscillatory reactions of many enzymes ${ }^{10}$. It was found that underlying cause of oscillatory reaction by enzyme was the suitable combination of permeation rate of substrate across membrane and rate constants of reaction. On the basis of these results, we considered that similar oscillation should occur in living system. In fact, we observed oscillations of NADH and ATP in mitochondria. It was thought that the oscillation started from the permeation of substrates through membrane in both cases of gradual entry of pyruvate and that of NAD and malate via malate-aspartate shuttle. Thus, we investigated the oscillatory reactions of enzymes existing in mitochondria by using dialysis membrane apart from investigating mitochondria. So, we reviewed results from these methods and compared.

The reaction of pyruvate, $\mathrm{NAD}^{+}$and $\mathrm{CoA}$ by pyruvate dehydrogenase utilizing dialysis membrane was investigated. In mitochondria, mitochondrial pyruvate transporter (MPC) makes the permeation of pyruvate, since pyruvate is not able to permeate through membrane ${ }^{14-15}$. The reaction with the aid of semipermeable membrane is considered to be valid as a simple model of the reaction with the permeation of substrates through naturally occurring biological membrane. NADH oscillation was observed in the reaction of pyruvate, $\mathrm{NAD}^{+}$and $\mathrm{CoA}$ by pyruvate dehydrogenase. This oscillatory reaction was considered to be caused by the gradual entry of pyruvate through dialysis membrane. The oscillatory reaction by pyruvate dehydrogenase has been investigated already, supporting our results ${ }^{15}$. Furthermore, the oscillation in the first stage of citric acid cycle was investigated and it was found that the oscillations of NADH and cis-aconitic acid occurred at the same time. It was suggested that the oscillation caused by gradual entry of pyruvate induced succeeding oscillatory reactions in citric acid cycle.

And then, we investigated the reactions of enzymes existing in the respiratory chain and discovered that the intermediates in respiratory chain, $\mathrm{NADH}$ and cytochrome $\mathrm{c}$ (reduced type), oscillated simultaneously with the same period. This suggests that the reactions in the respiratory chain also oscillate successively. Consequently, it would be expected that the NADH oscillation should also occur in the reactions via malate -aspartate shuttle within mitochondria.

As a comparison to these results, when isolated mitochondria were dispersed in pyruvate solution without ADP, the NADH oscillation was observed definitely. It is considered that the oscillatory reaction of pyruvate dehydrogenase, which is associated with the gradual entry of pyruvate through the mitochondrial inner membrane, induced consecutive oscillatory reactions in both of citric acid cycle and respiratory chain. At this condition ATP oscillation, however, did not appear yet. The addition of ADP in outer solution of mitochondria caused ATP oscillation. Also, as the supply of ADP led no oscillation in the 
absence of entry of pyruvate, both of the gradual entry of pyruvate and the supply of ADP, are thought to be essential to cause ATP oscillation. However, the NADH oscillation was not observed at the condition which the ATP oscillation occurred, being observed in higher concentration of pyruvate.

On the other hand, even when isolated mitochondria were dispersed in the solution of $\mathrm{NAD}^{+}$and malate, both oscillations of NADH and ATP were observed. Without the supply of ADP, ATP oscillation was not observed naturally. It was revealed that NADH produced in malate-aspartate shuttle ${ }^{19}$ oscillated and this reaction induced consecutive oscillatory reactions. For the same region of concentrations, we obtained not only oscillations of NADH, but also that of ATP as well.

We regarded that oscillation similar to oscillatory reaction in the use of semipermeable membrane should occur naturally in mitochondria. We could really obtain the oscillations of NADH and ATP in the reaction begin with the gradual entry of pyruvate or $\mathrm{NAD}^{+}$and malate by using mitochondria. The oscillation of $\mathrm{H}^{+}$ concentration was also observed. The process of respiratory chain creates an electrochemical proton gradient that drives the synthesis of ATP. The synthesis of ATP is carried out by ATP synthase existing in the inner membrane of mitochondria according to the density difference of $\mathrm{H}^{+}$transmembrane. As the oxidative phosphorylation is connected with the respiratory chain tightly, it is likely that oscillatory reactions occurring in respiratory chain induce the oscillation of ATP in oxidative phosphorylation.

The first description of mitochondrial oscillation was presented by Chance and Yoshioka and oscillations of ionic currents across mitochondrial membrane were explored ${ }^{17}$. On the other hand, it has been well known the oscillation of glycolysis as an oscillation in living system ${ }^{18-20}$. NADH and the other intermediate of glycolysis in the cytosol have been thought to oscillate with the aid of allosteric enzyme. Then, the oscillation of mitochondria was thought to result from the oscillation of glycolysis ${ }^{22-25}$. Apart from these, it had been also thought that reactive oxygen species (ROS) were involved in the oscillation of mitochondria ${ }^{26-28}$. Aon et al. made a hypothesis that balance between superoxide anion efflux through inner membrane anion channels and the intercellular ROS scavenging capacity play a key role in the oscillatory mechanism. Furthermore, the oscillation of mitochondria membrane potential and $\mathrm{Ca}^{2+}$ oscillation have been observed and the relationship with NADH and ATP oscillation has been being discussed $21,29-32$.

On the contrary, our findings demonstrated that the gradual entry of substrate caused the continuous oscillatory reactions of enzymes, which is conceivable to take place universally in living system. It was realized that oscillatory reaction, which was caused by gradual entry of first substrate in citric acid cycle or respiratory chain, induced the oscillations of NADH and ATP as well as pH oscillation. In addition, it was proved that oscillations of NADH and ATP occur within the isolated mitochondria unaffected by other.

We also be able to think that the oscillation of ion transport, the oscillation of mitochondria membrane potential and $\mathrm{Ca}^{2+}$ oscillation are thought to occur ancillary to the oscillatory reaction caused by gradual entry of substrate. The oscillation of mitochondria membrane potential can be thought of as results of 
$\mathrm{NADH}$ and the other intermediates oscillations via pH oscillation. We think that since there are many reactions involving membranes in the reaction in the living systems, it may be possible that many similar oscillations are present other than the mitochondria.

\section{Methods}

Sodium pyruvate, oxaloacetic acid, L-a- dipalmitoyl phosphatidylcholine (DPPC), Coenzyme $\mathrm{Q}_{10}$ (CoQ), hydrogen peroxide and L-malic acid were purchased from Wako Pure Chemical Industry, NAD ${ }^{+}, N A D H$ and ADP from Oriental Yeast Co., Ltd. and pyruvate dehydrogenase, Coenzyme A (CoA), citrate synthase, aconitase, cytochrome c, cytochrome $\mathrm{c}$ reductase, cytochrome $\mathrm{c}$ oxidase, luciferin and luciferase from Sigma-Aldrich Co..

Liposome solution was prepared as shown preceding paper ${ }^{11}$. At first, toluene containing $1 \mathrm{mM}$ DPPC and $10 \mathrm{mM} \mathrm{CoQ}$ was layered on the buffer solution of equal volume.

The transfer of DPPC was confirmed with the following method. Two days after laying the toluene solution of DPPC without CoQ on the aqueous solution, the toluene solution of DPPC was evaporated to dryness and the mass of DPPC remained was weighted. The result indicated that $86.4 \%$ of DPPC transferred into the aqueous solution from toluene solution. Likewise, concentration of remained CoQ in the toluene solution was determined by measuring the absorbance at $281 \mathrm{~nm}$. From this result, it was found that the concentration of $\mathrm{CoQ}$ transferred into the aqueous solution was $1.54 \mathrm{mM}$.

Apparatus for measuring the absorbance is shown in Fig. 3. Glass tube covered with dialysis membrane at the one end was inserted into a cuvette which contained enzyme solution $(2 \mathrm{~mL})$. Substrate solution $(1 \mathrm{~mL})$ was put into the glass tube. Cuvette was mounted in a brass block which was thermally controlled by circulating water at constant temperature, $37^{\circ} \mathrm{C}$. The time course of the absorbance (ABS) of lower phase was measured by spectrophotometer (Shimadzu UV-1800).

On the other hand, mitochondria were prepared using Mitochondria Isolation Kit for Tissue and Cultured cells of BioChain Institue Inc. from beef of $2 \mathrm{~g}$. The fluorescence intensity was measured by spectrofluorophotometer (Shimadzu RF-5300). In order to measure the $\mathrm{pH}$ within mitochondria, BCEAMAF (2',7'-bis-(2-carboxyethyl)-5-(and-6)-carboxyfluorescein, acetoxymethyl ester) was used as $\mathrm{pH}$ dependent fluorescence reagent ${ }^{33}$. Mitochondria solution was incubated in the presence of $0.1 \mathrm{mM}$ BCECF-AF for 30 min at $25^{\circ} \mathrm{C}$ and mitochondria including BCECF-AF were separated from external solution by centrifugation. Fluorescence intensity of BCECF was measured at the wavelength of $500 \mathrm{~nm}$ (exciting light) and $530 \mathrm{~nm}$ (emission light).

All aqueous solutions used were adjusted at $\mathrm{pH} 8$ with Tris buffer.

\section{References}


1. Nicolis, G. and Prigogine, I., Self-Organization in nonequilibrium systems From dissipative structures to order through fluctuation, John Willey and \& Sons,Inc. (1977)

2. Belousov, B. P., A periodic reaction and its mechanism, in Collection of short papers on radiation medicine for 1958, Med. Publ., Moscow (1959)

3. Field, R. J., Koros, E., and Noyes, R. M., Oscillations in chemical systems .2. Thorough analysis of temporal oscillation in bromate-cerium-malonic acid system, J. Amer. Chem. Soc.94, 8649-64 (1972)

4. Field, R. Noyes, R.M., Oscillations in chemical systems. IV. Limit cycle behavior in a model of a real chemical reaction. J. Chem. Phys. 60, 1877-1884 (1974)

5. M.Duysens, L. N, Amesz, J., Fluorescence spectrophotometry of reduced phosphopyridine nucleotide in intact cells in the near-ultraviolet and visible region. Biochimica et Biophysica Acta. 24 (1957)

6. Goldbeter, A., Biochemical Oscillations and cellular rhythms The molecular bases of periodic and chaotic behavior, Cambridge University Press (1996)

7. Hideshima, T. and Inoue, T., Biophys. Chem. 63, 81-86 (1997)

8. Hideshima, T., Oscillatory reaction of alcohol dehydrogenase in an oil/water system, Biophys. Chem. 38, 265-268 (1990)

9. Hideshima, T., Simulation of an oscillatory reaction of alcohol dehydrogenase in an oil/water system, Biophys. Chem.39, 171-175 (1991)

10. Hideshima, T., Science of non-equilibrium system VI, Seitai no Shindou Hannou (Oscillatory Reaction in Living Systems, Kodansha, 125-183 (2002)

11. Hideshima, T., Kato, Y., Biophys. Chem.124, 100-105 (2006)

12. Hideshima, T., Kobayashi, T.Araki, T. and Sasaki, S., Biochemical and Biophysical Research Communications 315, 119-122 (2004)

13. Lu, M., Zhou, L., Stanley, W. C., Cabrera, M. E., Saidel, G. M and Yu, X., Role of the Malate-Aspartate Shuttle on the Metabolic Response to Myocardial Ischemia, J. Theor. Biol. 254, 466-475

14. Bender, R., Martinou, J-C., The mitochondrial pyruvate carrier in health and disease: To carry or not to carry, Biochimica et Biophysica Acta, 1863, 2436-2442 (2008)

15. Vacanti, N. M., Divakaruni, A. S, Green, C. R., Parker, S. J., Henry, R. R., Ciaraldi, T. P, Murphy, A. N. and Metallo, C. M., The role of the mitochondrial pyruvate carrier in substrate regulation, Mol Cell. 56, 425-435 (2014)

16. Selivanov, V.A., Zakrzhevskaya, D.T. and Goldstein, B.N., Activity oscillations predicted for pyruvate dehydrogenase complexes, FEBS Letters 345, 151-153 (1994)

17. Chance, B. and Yoshioka, T., Sustained oscillations of ionic currents mitochondria, Arch.Biochem.Biophys., 117, 451-465 (1966)

18. Chance, B., Estabrook, R. W., Ghosh, A., Damped sinusoidal oscillations of cytoplasmic reduced pyridine nucleotide in yeast cells. Proc Natl Acad Sci USA. 51:1244-1251 (1964)

19. Hommes, F.A. and Schuurmansstekhoven, F.M., A periodic changes of reduced nicotinamide-adenine dinucleotide during anaerobic glycolysis in brewer's yeast. Biochim Biophys Acta. 86, 427-428 
(1964)

20. Olsen, L.F., Anderson, A. Z , Lunding, A., Brasen, J. C. and Poulsen, A. K., Regulation of Glycolytic Oscillations by Mitochondrial and Plasma Membrane H -ATPases, Biophys J. 96, 3850-3861 (2009)

21. Namekata, I., Kaeriyama, S., Hamaguchi, S. and lida-Tanaka, N., Mitochondrial membrane potentials oscillations in isolated cardiomyocytes as revealed by fluorescence microscopy, Current Topics in Pharmacology 20, 39-44 (2016),

22. Ainscow, E. A. and Rutter, G. A., Glucose-stimulated oscillations in free cytosolic ATP concentration imaged in single islet beta-cells: evidence for a $\mathrm{Ca}^{2+}$-dependent mechanism, Diabetes $51,62-70$ (2002)

23. Chandra, F. A., Buzi, G., and Doyle, J. C., Glycolytic Oscillations and Limits on Robust Efficiency, Science 333, 187- 192(2011)

24. Olsen, L. F., Andersen, A., Brasen, J. C. and Poulsen, A. K., Regulation of Glycolytic Oscillations by Mitochondrial and Plasma Membrane $\mathrm{H}^{+}$-ATPases, Biophys J, 96, 3850-3861 (2009)

25. Gooch, V and Packer, L., Oscillatory systems in mitochondria, Biochim. Biopys. Acta. 346, 245-260 (1974)

26. Cortassa, S., Aon, M.A., Winslow, R.L. and O'rouke, B.,A mitochondrial oscillator dependent on reactive oxygen species, Biophysical J. 87, 2060-2073 (2004)

27. Aon, V, Cortassa, V and O'Rourke, B., Mitochondrial oscillation in physiology and pathophysiology, Adv Exp Med Biol. 641, 98-117(2008)

28. Aon, M. A., Cortassa, S., Marbán, E. and O'Rourke, B., Synchronized Whole Cell Oscillations in Mitochondrial Metabolism Triggered by a Local Release of Reactive Oxygen Species in Cardiac Myocytes, The Journal of Biological Chemistry 273, 44735-44744 (2003)

29. Chalmers, S. and McCarron, J. G., The mitochondrial membrane potential and $\mathrm{Ca}^{2+}$ oscillations in smooth muscle, Journal of Cell Science 121, 75-85 (2007)

30. Chance, B. and Yoshioka, T., Sustained oscillations of ionic constituents of mitochondria, Archives of biochemistry and biophysics, 117,451-465 (1966)

31. Kwon, J., Omiya, Y., Homma, K I., Honma, S., Nagai, T., Saito, K. and Yasuda, Y. , Synchronized ATP oscillations have a critical role in prechondrogenic condensation during chondrogenesis, Cell Death Dis. 3, 278-289 (2012)

32. Andrei, I., Tarasova, I., Elinor, J, Griffiths, B.1. and Rutter, G. A., Regulation of ATP production by mitochondrial $\mathrm{Ca}^{2+}$, Cell Calcium 52, 28-35 (2012)

33. Kapus, A., Ligeti, E. and Fonyo, A., $\mathrm{Na}^{+} / \mathrm{H}^{+}$exchange in monitored by BCECF fluorescence, FEB 251, 49-52 (1989)

\section{Figures}


a
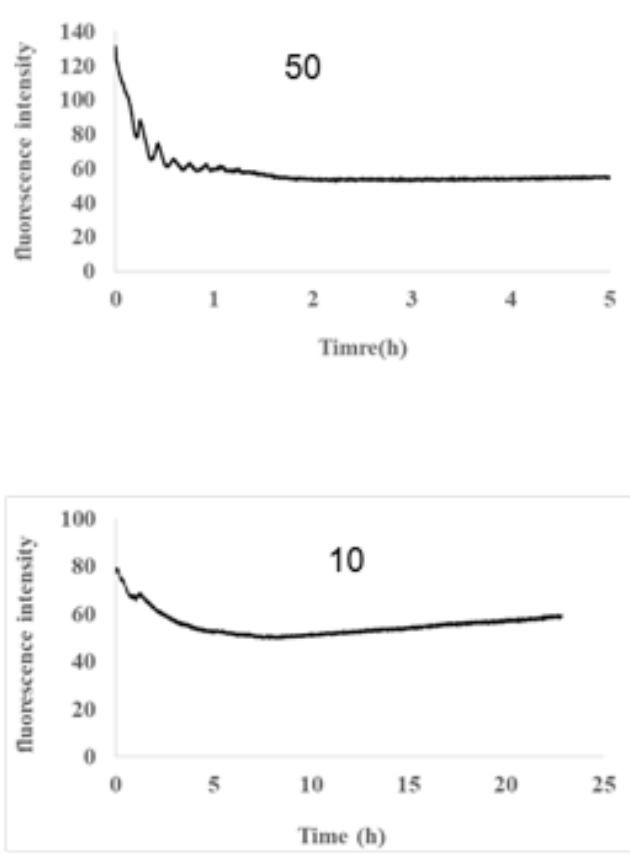

b
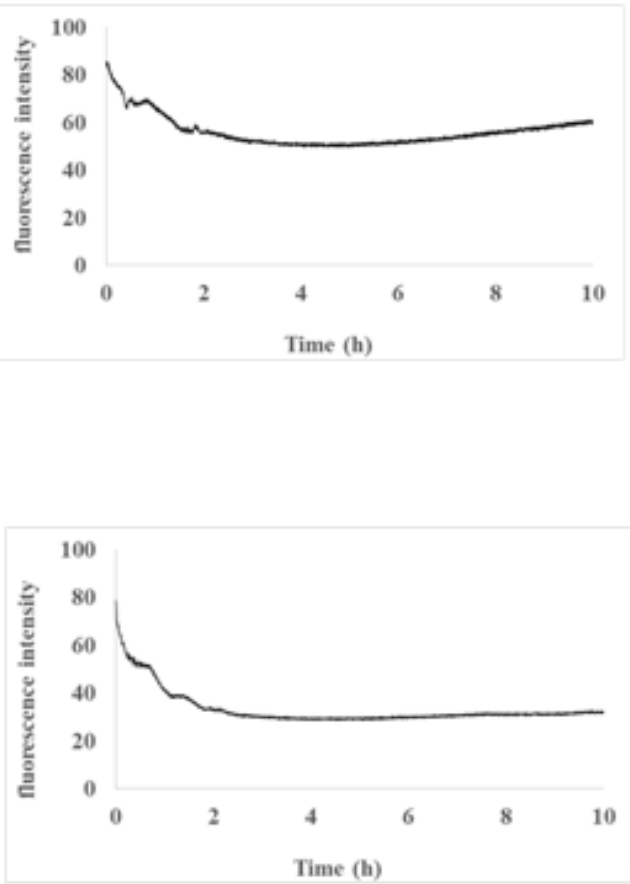

C
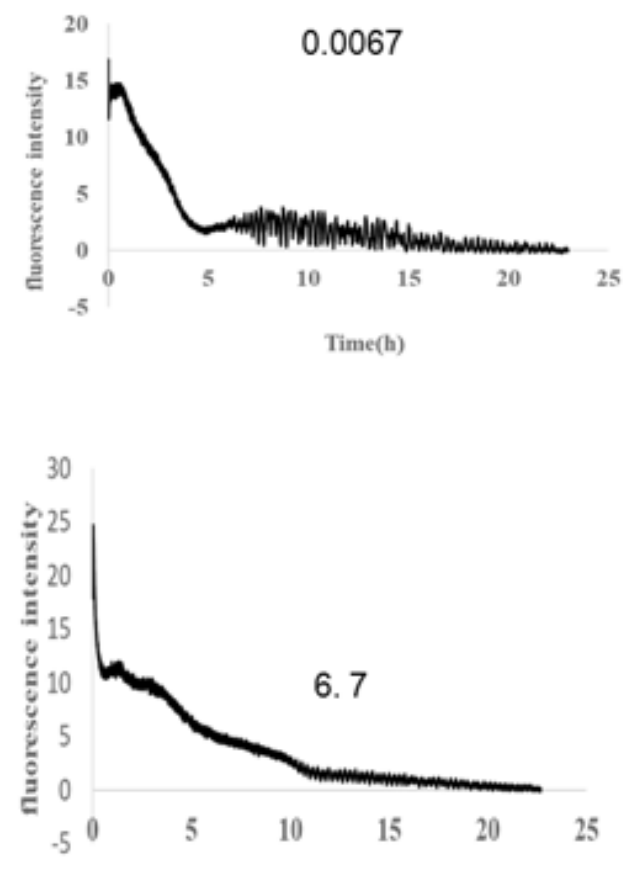

Time(h)

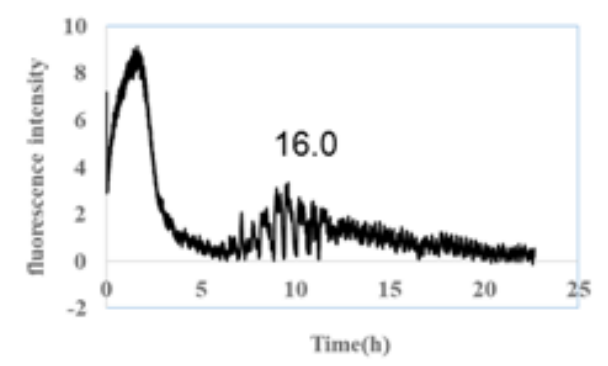

d

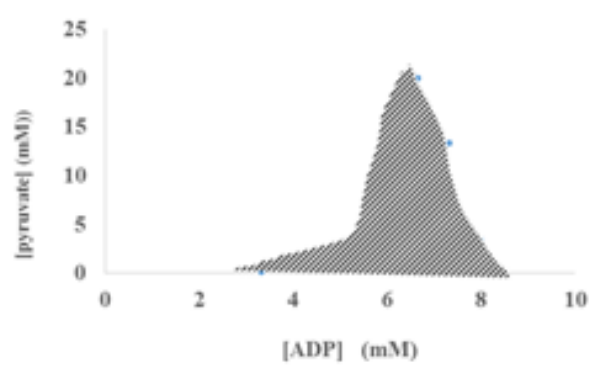

\section{Figure 1}

Oscillations of NADH and ATP in the case of gradual entry of pyruvate. a. Time course of fluorescence intensity due to NADH in case of the absence of ADP supply. Concentration of pyruvate is shown in the figure in mM. b. Time course of fluorescence intensity due to NADH in the presence of ADP supply. Above figure: $[$ pyruvate] $=10 \mathrm{mM}$ and $[A D P]=10 \mathrm{mM}$. Below figure: $[$ pyruvate $]=50 \mathrm{mM}$ and $[A D P]=6 \mathrm{mM}$. c. Time course of fluorescence intensity due to ATP for $[A D P]=6.67 \mathrm{mM}$. Concentration of pyruvate is 
shown in the figure in $\mathrm{mM}$. d. Phase diagram for oscillatory reaction. In below the curve line oscillatory reaction occur.

a
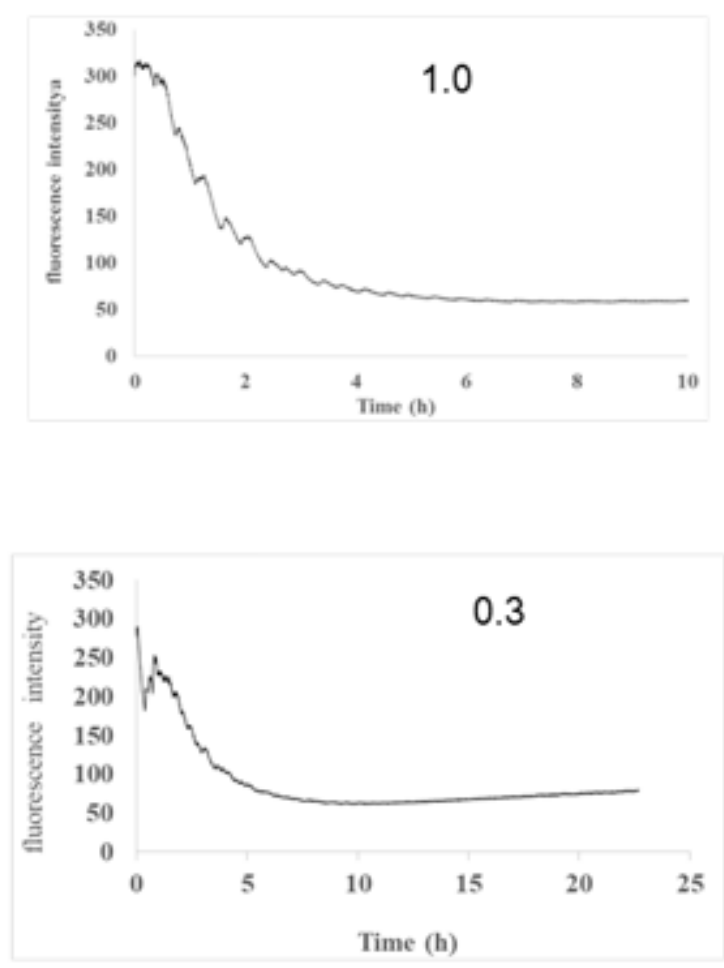

b

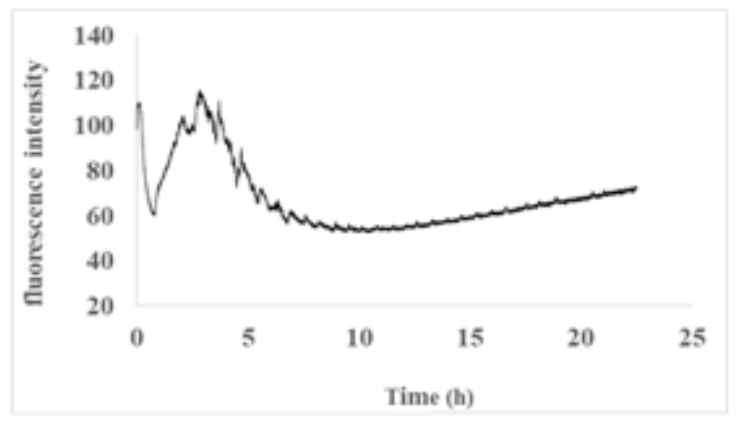

C
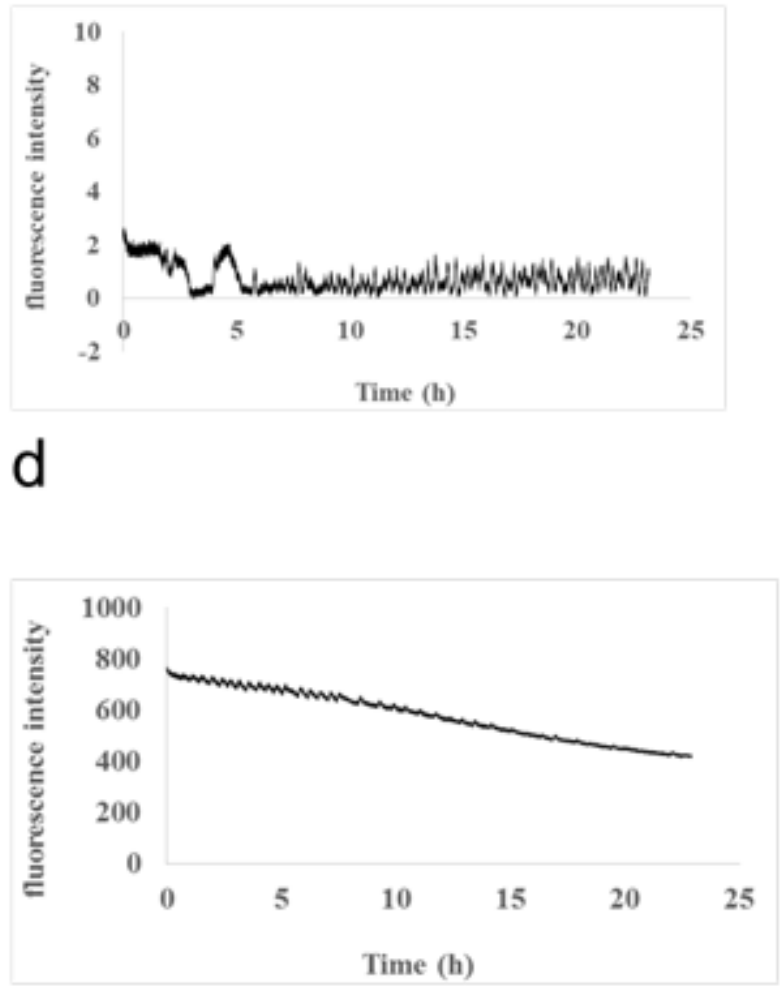

e

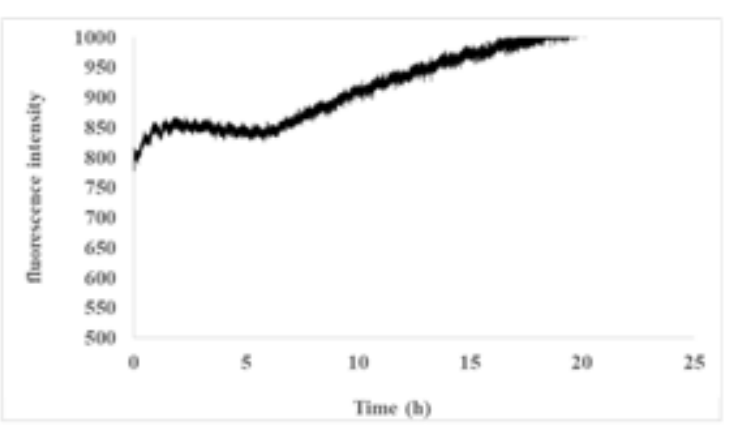

\section{Figure 2}

Oscillations of NADH and ATP in the case via malate-aspartate shuttle. a. Time course of fluorescence intensity due to NADH in the absence of ADP supply. Concentrations of NAD+ is shown in the figure in $\mathrm{mM}$. The concentration of malate is the same as that of NAD+. b. Time course of fluorescence intensity due to $\mathrm{NADH}$ in the presence of $\mathrm{ADP}$ supply. $[\mathrm{NAD+}]=2 \mathrm{mM}$, [malate] $=2 \mathrm{mM}$ and $[\mathrm{ADP}]=20 \mathrm{mM}$. c. Time course of fluorescence intensity due to ATP. $[\mathrm{NAD+}]=2 \mathrm{mM}$, [malate $]=2 \mathrm{mM}$ and $[A D P]=20 \mathrm{mM}$. $\mathrm{d}$. Time course of $\mathrm{pH}$-dependent fluorescence intensity within mitochondria in the permeation of pyruvate. [pyruvate] $=1 \mathrm{mM}$ and $[\mathrm{ADP}]=6 \mathrm{mM}$. e. Time course of $\mathrm{pH}$-dependent fluorescence intensity within 
mitochondria in the permeations of $\mathrm{NAD}+$ and malate. $[\mathrm{NAD}+]=2 \mathrm{mM}$, [malate] $=2 \mathrm{mM}$ and $[A D P]=$ $20 \mathrm{mM}$.

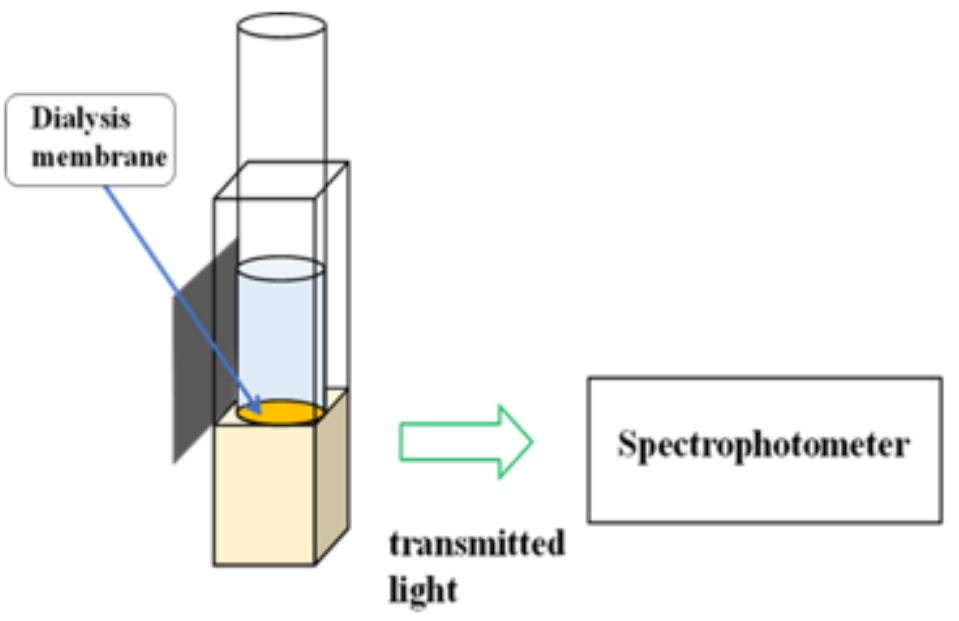

Figure 3

Apparatus for measuring the absorbance 
a
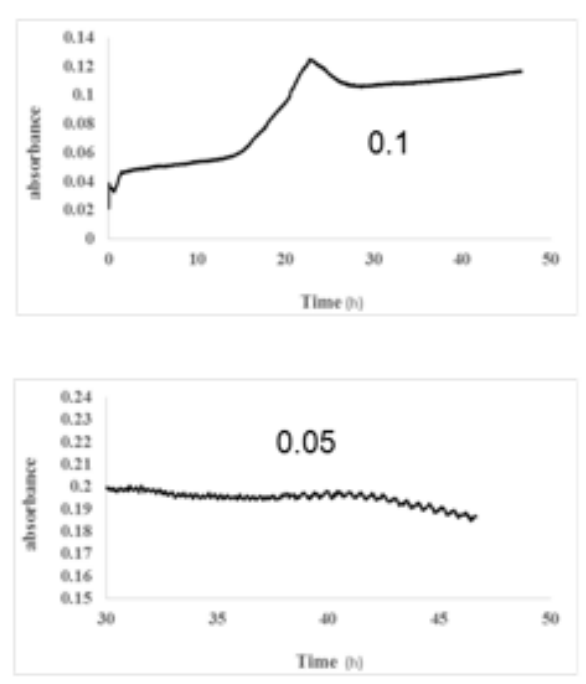

b
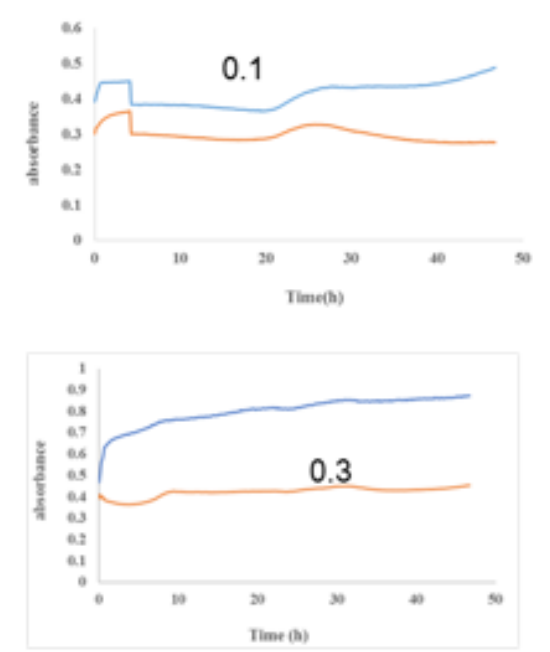

e
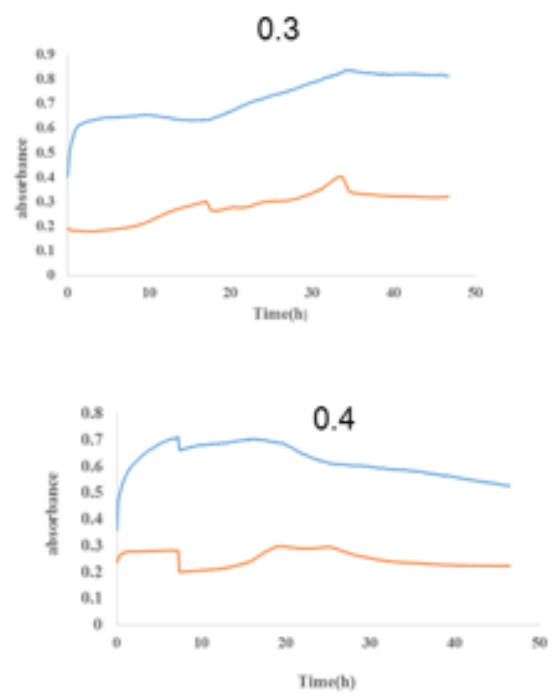

d
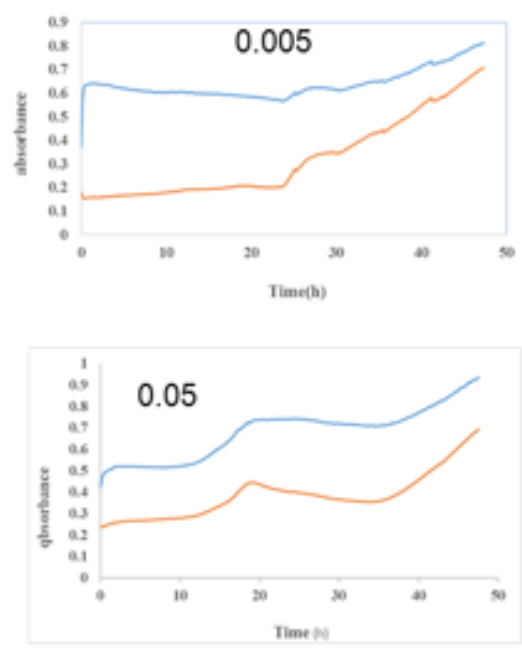

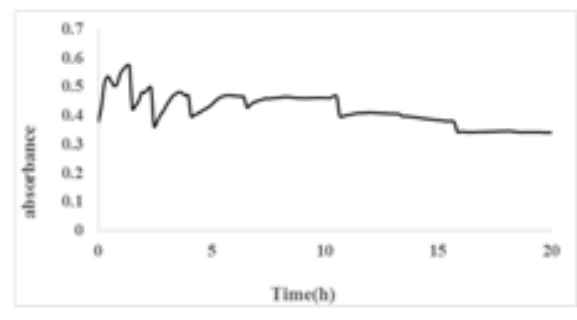

\section{Figure 4}

Results in the case using semipermeable membrane for reaction via malate-aspartate shuttle. a. Time course of absorbance in the reaction of NADH and CoQ. Concentration of NADH is shown in the figure in $\mathrm{mM}$. b. Oscillatory reaction of NADH and reduced cytochrome c. [cytochrome c] $=0.2 \mathrm{mg} / \mathrm{mL}$, [cytochrome $c$ reductase] $=0.05 \mathrm{mg} / \mathrm{mL}$. Blue and red lines indicate $\mathrm{NADH}$ and reduced type-cytochrome $\mathrm{c}$, respectively. Concentration of NADH is shown in the figure in mM. c. Oscillatory reactions of NADH and 
reduced cytochrome c. [cytochrome c] $=0.2 \mathrm{mg} / \mathrm{mL}$, [cytochrome $\mathrm{c}$ reductase] $=0.05 \mathrm{mg} / \mathrm{mL}$ and [cytochrome c oxidase] $=0.005 \mathrm{mg} / \mathrm{mL}$. Blue and red lines indicate $\mathrm{NADH}$ and reduced type-cytochrome c, respectively. The concentration of $\mathrm{NADH}$ is shown in the figure in $\mathrm{mM}$. d. Oscillatory reactions of NADH and reduced cytochrome. $[\mathrm{NADH}]=0.3 \mathrm{mM}$, [cytochrome $\mathrm{c}]=0.2 \mathrm{mg} / \mathrm{mL}$ and [cytochrome $\mathrm{c}$ reductase] $=0.005 \mathrm{mg} / \mathrm{mL}$. Blue and red lines indicate NADH and reduced type-cytochrome c, respectively. The concentration of cytochrome $\mathrm{c}$ oxidase is shown in the figure in $\mathrm{mg}$. e. Oscillatory reaction of NADH in the supply of oxygen.

\section{a}
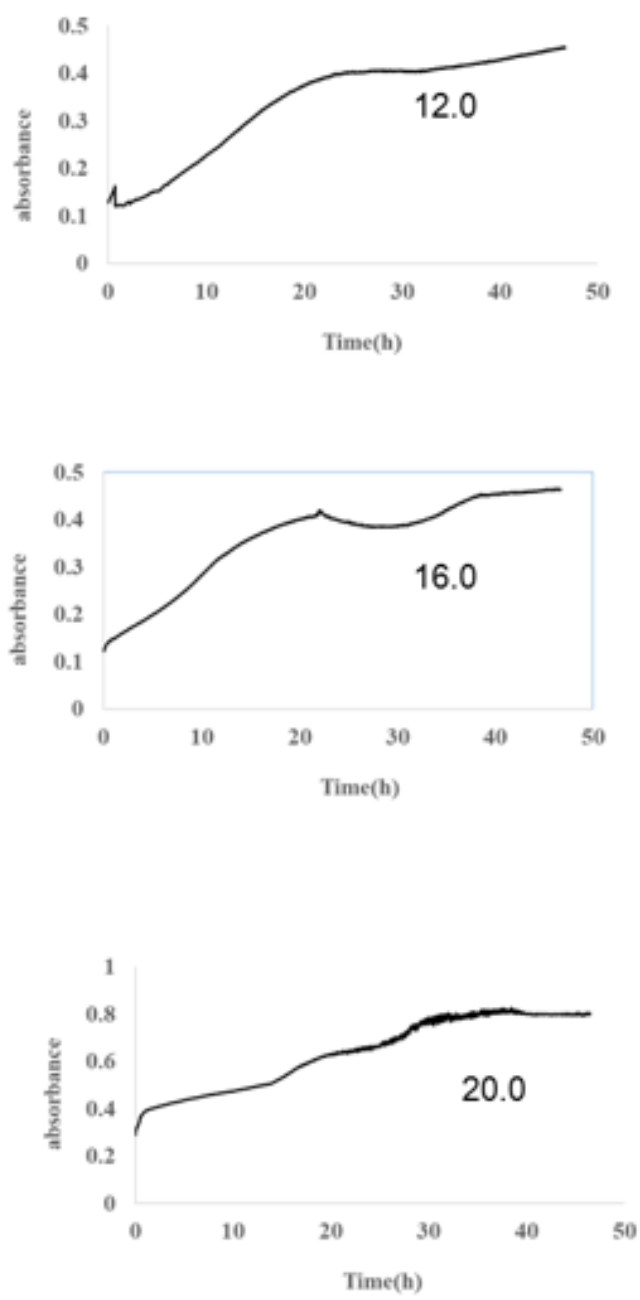

b

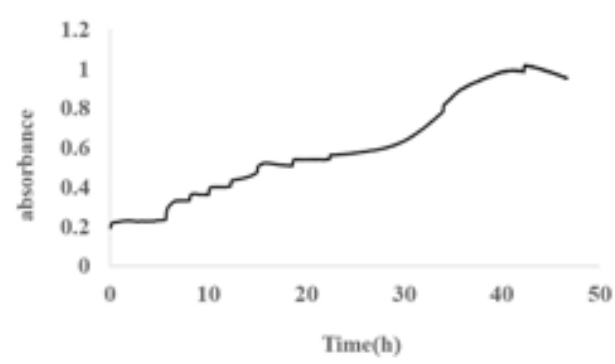

\section{C}
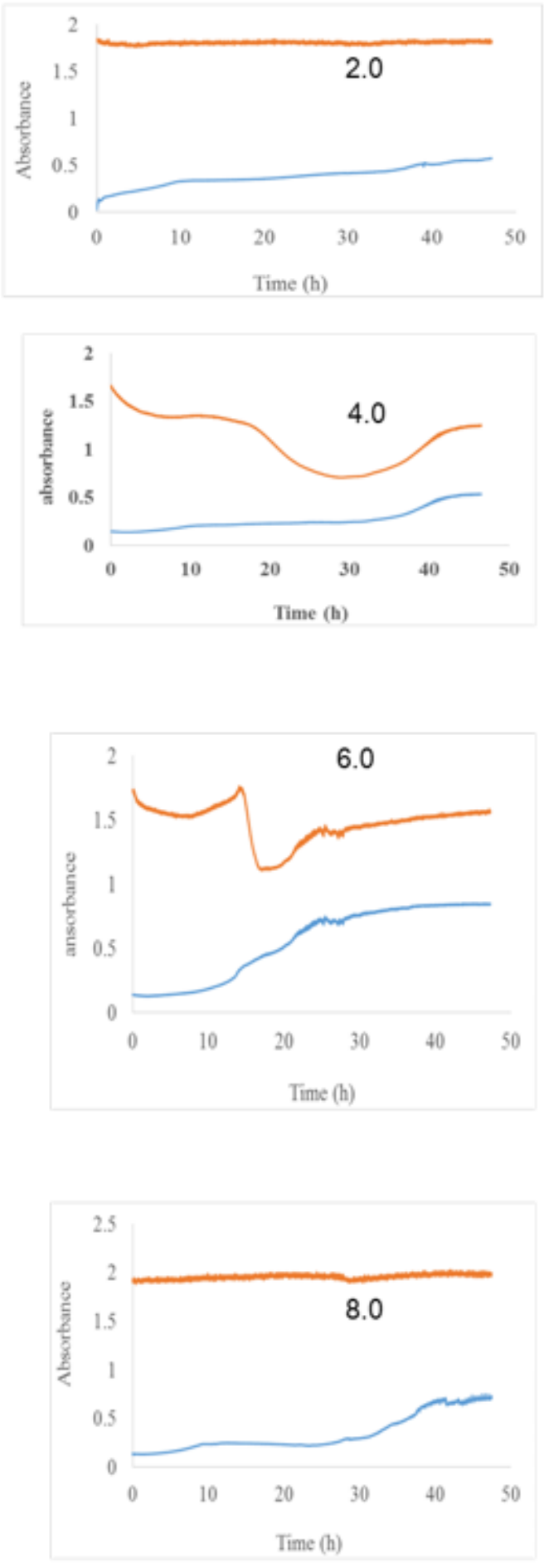


\section{Figure 5}

Results in the case using semipermeable membrane for gradual entry of pyruvate. a. Time course of absorbance. $[\mathrm{NAD+}]=0.25 \mathrm{mM}$, [pyruvate dehydrogenase $]=0.04 \mathrm{mg} / \mathrm{mL}$ and $[\mathrm{CoA}]=0.5 \mathrm{mM}$. Concentration of pyruvate is shown in the figure in $\mathrm{mM}$. b. Time course of absorbance of NADH and cis-aconitic acid. [pyruvate] $=8 \mathrm{mM},[\mathrm{NAD}+]=0.25 \mathrm{mM}$, [pyruvate dehydrogenase $]=0.04 \mathrm{mg} / \mathrm{mL}$ and $[\mathrm{CoA}]=0.05 \mathrm{mM}$. c. Time course of absorbances of NADH and cis-aconitic acid. [NAD+] $=0.25 \mathrm{mM}$, [pyruvate dehydrogenase] $=0.025 \mathrm{mg} / \mathrm{mL}$, [CoA] $=0.05 \mathrm{mM}$, [oxaloacetic acid] $=0.5 \mathrm{mM}$, [citrate synthase $=0.000115 \mathrm{mg} / \mathrm{mL}$ and [aconitase] $=0.000093 \mathrm{mg} / \mathrm{mL}$. The concentration of pyruvate is shown in the figure in $\mathrm{mmol} / \mathrm{L}$. Blue and red lines indicate NADH and cis-aconitic acid, respectively. 